\title{
Brevets et stratégies de valorisation de l'innovation
}

\section{Patents and the strategic approach to the valorisation of innovation}

\author{
Marc Baudry ${ }^{1}$, Beatrice Dumont ${ }^{2}$ \\ ${ }^{1}$ Université Paris Nanterre-CNRS \& Chaire Economie du Climat, France, marc.baudry@parisnanterre.fr \\ ${ }^{2}$ Université Sorbonne Paris Nord-CNRS \& College of Europe, France, beatrice.dumont@univ-paris13.fr
}

RÉSUMÉ. Ce numéro de Technologie et Innovation propose de mettre en regard les analyses disciplinaires, notamment celles issues de l'économie, de la finance, de la comptabilité et du droit, quant aux stratégies de valorisation de l'innovation et de leur lien, ou opposition, avec les brevets. II se veut donc fondamentalement pluridisciplinaire et, chemin faisant, cherche à promouvoir tant chez les chercheurs que chez les praticiens une vision globale des atouts et limites des brevets dans la construction d'une stratégie de valorisation de l'innovation.

ABSTRACT. This issue on the subject of Technology and Innovation proposes to compare disciplinary analyses, particularly those from economics, finance, accounting and law, on strategies for enhancing the value of innovation and their link, or opposition, to patents. It is therefore fundamentally multidisciplinary and, along the way, seeks to promote among both researchers and practitioners a global vision of the advantages and limitations of patents in the construction of a strategy for the valorisation of innovation.

MOTS-CLÉS. Propriété intellectuelle, Brevets, Stratégie, Economie, Droit, Finance, Comptabilité.

KEYWORDS. Intellectual property, Patents, Strategy, Economics, Law, Finance, Accounting.

La propriété intellectuelle, et plus singulièrement les brevets, ont vu leur utilisation fortement évoluer au cours des dernières décennies [BAU 17]. Sans abandonner leur rôle de rempart destiné à sécuriser les gains tirés de l'exploitation industrielle et commerciale de l'invention brevetée, l'utilisation plus «stratégique » des brevets s'est accrue, notamment sous la forme de brevets bloquants, voire un rôle jugé souvent pernicieux dans le cadre du modèle d'affaires des patent trolls. Toutefois, ces derniers, en centrant leur activité sur l'assertion, auprès des tribunaux, de brevets qu'ils ont acquis ou des brevets d'entreprises clientes, renforcent aussi l'attention portée par chaque entreprise innovante à ne pas enfreindre les innovations brevetées par leurs concurrentes. Les brevets ne se limitent plus, en outre, à la relation avec les concurrents mais ont largement investi également le domaine financier. Grâce, par exemple, à leur fonction de signal crédible de la capacité inventive des inventeurs, ils peuvent faciliter l'accès au capital risque ou l'introduction en bourse de la firme détentrice. Ils ont également permis l'émergence d'un marché des technologies et favorisé en cela le développement des fabless. En conciliant protection et divulgation des inventions, ils contribuent ainsi notoirement à l'essor de l'open innovation. Il résulte de ces évolutions que les décisions stratégiques qu'une entreprise est amenée à faire en matière de brevets sont multiples. Sans prétendre à l'exhaustivité, ce numéro vise à les mettre en lumière par différentes contributions venues de champs disciplinaires couvrant la comptabilité, la finance, le droit et l'économie.

Un brevet est incontestablement pour son détenteur un actif au sens économique du terme, c'està-dire qu'il produit et conserve à travers le temps de la valeur, ou tout au moins il véhicule une espérance de valeur. Il n'est pas pour autant aisé d'apporter des éléments probants de la réalité et du montant de cette valeur. Bien que constituant un actif au sens économique du terme, un brevet ne satisfait donc pas nécessairement les critères pour que la valeur qu'il représente pour l'entreprise qui le détient puisse aisément figurer tout ou partie à l'actif de son bilan. Comme l'explique Lionel Touchais dans la première contribution, les normes comptables imposent une règle de prudence qui rend généralement difficile l'activation de la valeur des brevets au bilan de l'entreprise. Ces règles diffèrent toutefois selon la norme comptable utilisée, norme nationale française, norme nationale américaine ou encore norme internationale IFRS. La possibilité d'activation dépend aussi du mode d'acquisition du brevet, notamment selon qu'il est produit en interne ou acquis en externe. La 
révision de la valeur au bilan obéit également à des règles précises. Les contraintes d'activation, inhérentes aux principes de base de la comptabilité, couplées à l'hétérogénéité des règles selon la norme comptable utilisée, ainsi quun certain degré de liberté laissé quant au choix d'activer ou pas ce qui peut l'être, sont autant de facteurs qui créent une asymétrie d'information entre le ou les manager(s) de l'entreprise et les investisseurs (potentiels). Cette asymétrie joue en défaveur de l'investissement en R\&D et donc des entreprises innovantes. Certaines stratégies de communication extra financière peuvent au moins en partie réduire l'asymétrie d'information de manière efficace. En procédant à une revue de la littérature des études ayant cherché à tester l'impact des choix d'activation de brevets et des choix de communication hors bilan sur la valeur de marché des entreprises, Lionel Touchais conclut que ces choix ne sont pas neutres. L'information hors bilan que peut produire une entreprise sur ces brevets doit être la plus objective et crédible possible pour que l'effet de signal auprès des investisseurs fonctionne. Cette information va donc souvent s'appuyer sur ce que la littérature nomme les métriques de brevets, c'est-à-dire des éléments facilement quantifiables et vérifiables qui figurent dans les registres de brevets tenus par les offices de brevets. Une métrique couramment utilisée pour donner ainsi un éclairage sur l'importance d'un brevet est le nombre de citations faites de ces brevets par des brevets plus récents. Les investisseurs peuvent ne pas se contenter de l'information hors bilan produite par les entreprises et chercher à exploiter eux même directement l'information fournie par les offices de brevets. Dans les deux contributions suivantes, Didier Lebert et François-Xavier Meunier tout d'abord, puis Valentin Lignau, s'intéressent respectivement aux classes technologiques et aux oppositions pour mettre en lumière ce que l'exploitation de ces informations peut révéler quant à la stratégie des entreprises d'un secteur.

Didier Lebert et François-Xavier Meunier explorent dans leur contribution les choix stratégiques effectuées par les entreprises en matière de $R \& D$ entre activité d'exploitation et activité d'exploration. Selon la distinction faite par [MAR 91], l'invention exploratoire exige de battre en brèche les domaines de compétences maîtrisées par l'entreprise, alors que l'invention d'exploitation s'appuie sur ses routines éprouvées. Dit autrement, l'exploitation vient renforcer les (processus de production de) connaissances dominantes - et les inventions produites sont de nature incrémentale alors que les inventions radicales nécessitent une transformation significative des processus et des productions routinières et sont donc issues de l'exploration. Dans leur contribution à ce numéro, Didier Lebert et François-Xavier Meunier appréhendent ces concepts d'exploitation versus exploration à partir de la Classification Internationale des Brevets (CIB) à quatre digits. L'idée sousjacente est que le portefeuille de brevets déposés par une entreprise auprès des différents offices de brevets à travers le mode reflète les connaissances développées par cette entreprise. Partant des classes CIB visées par les brevets d'une entreprise, ils expliquent comment construire des indices mesurant si l'entreprise cherche plutôt à se concentrer sur un cœur de classes technologiques, ou si elle n'hésite pas au contraire à s'aventurer vers des classes technologiques dont elle est peu coutumière. Les auteurs ne se limitent pas à une utilisation descriptive de ces indicateurs d'exploitation versus exploration. L'un des intérêts de leur contribution est qu'ils cherchent également à identifier les déterminants du choix des 2000 entreprises les plus innovantes à travers le monde de s'orienter plutôt vers l'exploitation ou plutôt vers l'exploration. Leur modèle économétrique teste notamment si l'étendue technologique de l'entreprise, sa centralité dans le réseau de relations entre classes technologiques qui émerge des brevets, la localisation de son siège social par rapport au marché considéré, parmi les trois marchés que couvrent respectivement l'Office Européen des Brevets (OEB,) l'US Patent and Trademark Office (USPTO) et le Japanese Patent Office (JPO), ont une influence statistiquement significative sur le choix opéré. Le type de choix analysé par Didier Lebert et François-Xavier Meunier peut être déterminant pour le succès de l'entreprise et la valeur qu'elle parviendra in fine à créer à partir de son portefeuille de brevets. Il n'en reste pas moins que tout brevet reste un pari. C'est un pari du fait qu'intrinsèquement les inventions brevetées visent des marchés nouveaux, certes de manière plus ou moins radicale, mais tout de même des marchés dont on n'est jamais certain de l'état exact de la demande avant de s'être (C) 2020 ISTE OpenScience - Published by ISTE Ltd. London, UK - openscience.fr 
lancé. Mais un brevet est également un pari du fait de l'incertitude juridique qui l'entoure. Certains spécialistes de l'étude du système des brevets, tels notamment [LEM 04], ont été amenés à parler de probabilistic patents tant l'incertitude juridique autour des brevets leur semble forte. De ce point de vue, le fait qu'un brevet survive à l'épreuve d'un litige est un signal fort de sa solidité juridique à partir duquel une entreprise peut, comme suggéré supra en présentant la contribution de Lionel Touchais, communiquer afin de réduire l'asymétrie d'information des investisseurs. Il en est de même des brevets qui passent avec succès l'épreuve des oppositions. Dans sa contribution, Valentin Lignau montre que l'étude des oppositions permet d'aller encore plus loin dans l'analyse des interactions stratégiques entre entreprises d'un secteur.

Si les litiges sur les brevets sont des procédures judiciaires postérieures à l'octroi d'un brevet, notamment les procès en contrefaçon, sur lesquelles statuent les tribunaux à défaut d'accord à l'amiable ou d'arbitrage privé entre les parties prenantes, les oppositions constituent quant à elle une procédure administrative, auprès de l'office des brevets et dans un délai relativement court après la publication du brevet. Une procédure d'opposition permet de contester que la demande de protection d'une invention répond au critère de brevetabilité. La procédure d'opposition intervient donc plus en amont dans la vie du brevet que le litige. Surtout, parce qu'elles sont traitées par l'office de brevet, l'information sur les oppositions et leur issue est communiquée de manière systématique et est donc nettement plus accessible que celle sur les litiges. Une opposition mettant en rapport, comme du reste un litige, au moins deux firmes dont l'une est détentrice du brevet opposé, peut être révélatrice d'une utilisation stratégique de la propriété intellectuelle par une firme ou plus globalement par les firmes d'un secteur. Si, par exemple, il s'avérait que statistiquement une firme voit ses oppositions à l'encontre des brevets déposés par ses concurrents significativement plus échouer que la moyenne des oppositions dans le secteur, cela peut être le signe que cette firme utilise stratégiquement la procédure. En effet, ce qu'elle est susceptible de rechercher n'est peut-être pas tant la révocation ou l'amendement du brevet opposé mais plutôt le surcoût financier et le délai d'introduction de l'invention sur le marché imposés au concurrent. De même, s'il s'avérait que les grands acteurs d'un secteur intentent statistiquement plus d'oppositions vis-à-vis de petites firmes tentant d'entrer sur le marché que vis-à-vis des autres grands acteurs du secteur, alors cela peut être le signe d'un comportement de prédation et d'érection de barrières à l'entrée.. C'est ce type de comportements stratégiques que Valentin Lignau cherche à déceler dans sa contribution, en ciblant plus spécifiquement le secteur des turbines éoliennes en Europe. En effet, comme illustré par Valentin Lignau, ce secteur a connu un développement marqué sur les vingt dernières années, tant en terme de dynamique des installations que d'évolution technique des modèles proposés. Ces évolutions se sont accompagnées d'une modification de la structure de marché, avec une baisse du nombre d'acteurs et une hausse de la concentration des parts de marché. La phase de maturité que le marché semble avoir atteint peut être propice à un recours accru à la propriété intellectuelle, non plus comme outil de protection d'inventions alimentant la croissance du marché, mais comme arme de nuisance voire de dissuasion à l'encontre de concurrents en place ou des entrants potentiels. Valentin Lignau met en évidence que, dans le secteur des turbines éoliennes en Europe, le basculement général vers une telle utilisation stratégique des oppositions ne semble pas avoir été opérée. La pratique des oppositions y semble principalement régie par la volonté de contester, de bonne foi, la brevetabilité des inventions ainsi visées. Il n'en reste pas moins que le comportement de certains acteurs a changé, avec un recours accru aux oppositions au moins corrélé, si ce n'est causé, par un effritement marqué des parts de marché. A l'inverse certains acteurs ont pu voir les oppositions à l'encontre de leurs brevets croître à mesure qu'ils gagnaient des parts de marché. Si elle reste marginale, l'utilisation stratégique des oppositions n'est donc pas totalement inconnue dans ce secteur. L'analyse des oppositions faite par Valentin Lignau et le fait qu'elles peuvent être utilisées de manière agressive à l'encontre des concurrents, dépassant en cela le rôle de protection des inventions et d'incitation à l'innovation originellement visé par le mécanisme des brevets, ne doit pas laisser à penser que l'évolution récente dans l'utilisation des brevets est dominé par les pratiques agressives. Bien au contraire, le développement de stratégies coopératives est un marqueur (c) 2020 ISTE OpenScience - Published by ISTE Ltd. London, UK - openscience.fr

Page $\mid 3$ 
fort de cette évolution récente à travers ce qu'il est convenu d'appeler l'innovation ouverte. C'est ce que mettent en exergue les deux dernières contributions de Nicolas Binctin d'une part et de Marc Baudry et Béatrice Dumont d'autre part, sous des angles différents.

Théorisée et popularisée par l'ouvrage de [CHE 03], l'innovation ouverte stipule qu'il peut être plus intéressant pour une entreprise d'être en interaction avec d'autres acteurs pour avancer dans ses propres projets innovants plutôt de se retrancher derrière des barrières étanches vis-à-vis de l'extérieur. Les partenaires vers lesquels l'entreprise est susceptible de s'ouvrir dans ce cadre sont soit des acteurs en amont ou aval, notamment des fournisseurs ou des clients, soit des concurrents, soit des structures académiques davantage positionnées sur la recherche fondamentale. Selon la forme d'ouverture, ces partenaires ont pu être pré-identifiés ou au contraire se révéler à travers le processus d'ouverture. Les formes d'ouverture sont assez diverses. Elles recouvrent l'externalisation de certaines parties de l'innovation, lorsque cette innovation n'est pas au cœur des compétences de l'entreprise, par exemple la construction de partenariats de $R \& D$, l'ouverture de l'accès aux innovations de l'entreprise et leur exploitation par d'autres avec ou sans conditions. Parfois réduite à de l'innovation «libre» (sous-entendu «libre de droits de propriété intellectuelle»), les études académiques tendent au contraire à montrer que l'innovation ouverte est loin d'être antinomique avec les brevets. En effet, un prérequis pour s'ouvrir est souvent d'une part de communiquer sur ce sur quoi on sait innover et, d'autre part, de protéger ce sur quoi on a déjà innové pour précisément s'ouvrir sans se faire spolier. Or les brevets répondent à ces deux exigences, protégeant par un droit d'exclusivité tout en diffusant par la publication. Un des aspects originaux de l'innovation ouverte est sans doute qu'elle peut se faire vis-à-vis de concurrents. Par contraction des termes anglais cooperation et competition, elle est alors désignée sous le vocable de coopetition, francisé en coopétition comme dans le titre de la contribution de Nicolas Binctin. S'ouvrir aux concurrents autour de projets d'innovation par essence stratégiques pour l'avenir de l'entreprise n'est pas sans poser des questions juridiques d'importance. C'est ce que discute Nicolas Binctin en se concentrant notamment sur deux aspects. Le premier aspect est l'évolution des contrats de coopération, plus précisément de leur gestion. L'auteur introduit notamment les concepts de comité de suivi et de contrat manager. L'un et l'autre offrent une agilité dans la gestion contractuelle des projets de coopération. Au risque de faire un raccourci, le premier permet de faire face aux imprévus et aux opportunités en lui déléguant explicitement les arbitrages et choix inhérents sans recourir incessamment à des avenants au contrat, tandis que le second permet d'être proactif dans la coopération en mettant en œuvre et coordonnant les moyens et processus nécessaires à la maitrise des risques encourus et à la saisie d'opportunités potentielles. Le second aspect discuté par Nicolas Binctin est la multiplicité des moyens juridiques de mettre en forme une coopétition. Trois moyens sont détaillés. Le premier est la gestion du portefeuille de droits d'exploitation, par la concession ou au contraire l'obtention de licences d'exploitation de brevets ; le second est la copropriété de brevets concrétisée sous la forme de co-brevets; le troisième est la mise en place d'une joint-venture. Les accords de licence peuvent se faire avec des partenaires qui n'avaient pas nécessairement été identifiés en amont du processus de $\mathrm{R} \& \mathrm{D}$, tandis les co-brevets résultent au contraire d'une coopération entre des partenaires que se sont rapprochés en amont de ce processus. Les accords de type joint-venture s'avèrent quant à eux adéquats dans le cadre d'une coopération asymétrique entre, d'une part, une entreprise bien établie et disposant de moyens financiers mais pas de la réactivité et/ou la souplesse requises pour innover dans un domaine qui n'est pas au cœur de ses compétences et, d'autre part, une jeune et généralement petite entreprise dont l'ADN est d'innover dans des domaines encore peut explorés mais qui souffre de la faiblesse de ses moyens de financement. En complément à la contribution de Nicolas Binctin et sous la forme d'une étude de cas tirant parti de l'information contenue dans les brevets, Marc Baudry et Béatrice Dumont explorent une autre stratégie d'ouverture, celle des patent pledges.

Le 12 juin 2014 Elon Musk, Directeur Général de la firme californienne Tesla, annonçait très médiatiquement au nom de la société qu'il dirige «All our patent are belong to you » (consultable 
sur le site de Tesla à l'adresse https://www.tesla.com/blog/all-our-patent-are-belongyou?redirect=no). Dans le même communiqué Elon Musk allait jusqu'à remettre en cause une stratégie d'innovation construite sur les brevets : "Technology leadership is not defined by patents, which history has repeatedly shown to be small protection indeed against a determined competitor, but rather by the ability of a company to attract and motivate the world's most talented engineers. ». Pourtant, comme le mettent en évidence Marc Baudry et Béatrice Dumont dans leur contribution, Tesla n'en a pour autant abandonné les demandes de brevets à partir de la date de cette annonce. Pourrait-il ainsi y avoir une rationalité à breveter pour ne pas exercer son droit d'exclusivité ? Ce type de stratégie relève des patent pledges, c'est-à-dire de l'engagement du détenteur d'un portefeuille de brevets à renoncer à tout ou partie de son droit d'exclusivité sur ce portefeuille ou un sous ensemble de ce portefeuille. Cet engagement peut se faire pour une durée limitée ou illimitée. Il se fait généralement sans contreparties financières, ou alors ces contreparties sont faibles ; le cas de licences FRAND étant parfois inclut dans la catégorie des patent pledges. Il peut également se faire avec des contreparties non financières, notamment sous la forme de licences croisées, ou sans la moindre contrepartie. Comme le soulignent Marc Baudry et Béatrice Dumont, le cas du patent pledge de Tesla est particulièrement intéressant à plusieurs égards. Tout d'abord, la firme Tesla s'est engagée de manière illimitée dans le temps ou sur l'intégralité de son portefeuille, quasiment sans la moindre contrepartie. Ensuite, cet engagement est pris par une entreprise récemment entrée sur un marché, celui de l'automobile, dominé par quelques gros acteurs implantés de longue date et réputé être dans une phase de renforcement de la concentration afin de relever le double défi de l'innovation dans le véhicule propre et dans le véhicule autonome. Pour comprendre en quoi le patent pledge de Tesla peut être profitable à la firme californienne, Marc Baudry et Béatrice Dumont commencent par analyser les parts de marché pour les véhicules tout électriques, puis comparent les portefeuilles de brevets des constructeurs sous différents angles. Les auteurs poursuivent leurs investigations en comparant la dynamique du portefeuille de brevets de Tesla à celle des portefeuilles de ses concurrents et concluent à l'absence de rupture significative dans cette dynamique après l'annonce du patent pledge. Battant en brèche l'idée d'un renoncement total aux brevets de la part de Tesla, ce résultat suggère plutôt une stratégie visant à imposer son leadership technologique. C'est ce qu'argumentent Marc Baudry et Béatrice Dumont en comparant, d'une part, le degré d'implication des constructeurs automobiles dans les coopérations en amont du processus de $R \& D$, telles que révélées par les co-brevets, et d'autre part le nombre de citations reçues par les brevets de Tesla par rapport à ceux de ces concurrents.

\section{Références}

[BAU 17] BAUDRY M., DUMONT B., Patents: prompting or restricting innovation?, Wiley - ISTE, Smart innovation SET, coordinated by D. Uzunidis, Londres, 2017.

[CHE 03] CHESBROUGH H., Open innovation: the new imperative for creating and profiting from technology, Harvard Business School Press, Boston, 2003.

[LEM 04] LeMLEY M. A., SHAPIRO C., «Probabilistic patents », Journal of Economic Perspectives, vol. 19(2), p. 7598, 2005.

[MAR 91] MARCH, J.G., «Exploration and exploitation in organizational learning ». Organization Science 2, Special Issue: Organizational Learning: Papers in Honor of (and by) James G. March, p. 71-87, 1991. 